Kansas State University Libraries

New Prairie Press

Conference on Applied Statistics in Agriculture 2010 - 22nd Annual Conference Proceedings

\title{
Editor's Preface and Table of Contents
}

Weixing Song

Follow this and additional works at: https://newprairiepress.org/agstatconference

Part of the Agriculture Commons, and the Applied Statistics Commons

\section{c) $($ ) $@ \Theta$}

This work is licensed under a Creative Commons Attribution-Noncommercial-No Derivative Works 4.0 License.

\section{Recommended Citation}

Song, Weixing (2010). "Editor's Preface and Table of Contents," Conference on Applied Statistics in Agriculture. https://doi.org/10.4148/2475-7772.1056

This is brought to you for free and open access by the Conferences at New Prairie Press. It has been accepted for inclusion in Conference on Applied Statistics in Agriculture by an authorized administrator of New Prairie Press. For more information, please contact cads@k-state.edu. 


\section{Conference Proceedings}

\section{Applied Statistics}
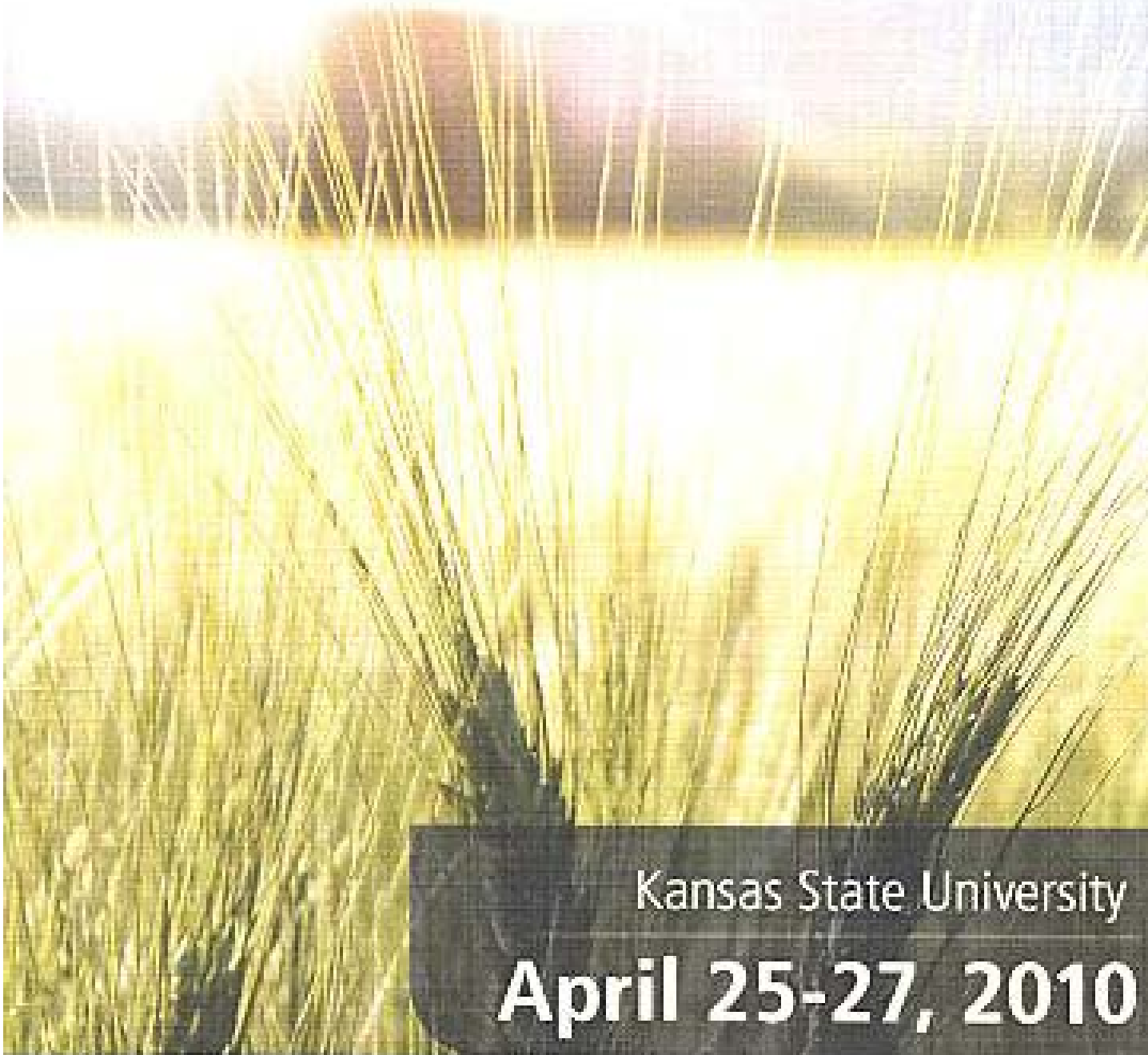


\section{Preface}

These proceedings contain papers presented in the twenty-second annual Kansas State University Conference on Applied Statistics in Agriculture, held in Manhattan, Kansas, April 25 - April 27, 2010. The purpose of the conference is to provide a forum for discussion on the application of statistics to problems in agricultural sciences. Papers and posters presented at the conference and included in the proceedings are intended to provide information to both the statistician and agricultural researcher on current issues of agricultural and general statistical interest. More than one hundred registrants for this year's conference came from across the country and represented government, industry, and academia.

The keynote speaker was Susanne May, Ph.D., University of Washington, Seattle. Dr. Susanne May presented a workshop on April 25 titled “Applied Survival Analysis”.

Each contributed paper was refereed before being accepted for publication in these proceedings. The papers were accepted for publication based on their interest, relevance, innovation and application to the agricultural sciences and statistics.

Sincere thanks to the following individuals: Joshua Tebbs, Reid Landes, Paul Auer, Kathy Yeater, Leigh Murray, Radha Mohanty, Jianming Yu, Tilman Achberger, Dennis Lock, Ed Gbur, Philip Dixon, Robert Foster, Chensong Zhu, Nora Bello, David Smith and Amy Tentiger for referring submitted manuscripts, Pam Schierer and student volunteers for help in conference planning and organization, and Angela Ladner for managing the reviewing process and for assembling the proceedings. Special thanks go to our conference sponsors for their continued support: American Statistical Association, Eli Lilly \& Company, Pfizer Inc, Pioneer Hi Bred-a Dupont Business and the USDA-ARS.

Weixing Song, Ph.D. 2010 Proceedings Editor
Gary L. Gadbury, Ph.D

Past Proceedings Editor 


\section{Table of Contents}

Modeling Fish Length Distribution Using A Mixture Technique

Bahman Shafii, University of Idaho

William J. Price, University of Idaho

Charlie Holderman, Kootenai Tribe of Idaho, Bonners Ferry, Idaho

Cathy Gidley, Idaho Department of Fish and Game, Coeur d'Alene, Idaho

Paul J. Anders, Cramer Fish Sciences, Moscow, Idaho

Characterizing Foraging Patterns among Cattle and Bonded and Non-Bonded Small Ruminants using Spatial Point Process Techniques

D.M. Anderson, U.S. Department of Agriculture, Las Cruces, NM

L.W. Murray, Kansas State University

P.Sun, GlaxoSmithKline, North Carolina

E.L. Fredrickson, U.S. Department of Agriculture, Las Cruces, NM

R.E. Estell, U.S. Department of Agriculture, Las Cruces, NM

V.B. Nakamatsu, Inta, Chubut, Argentina

Evaluating Pen-Day Interactions In Body Temperature Bilogistic

F. Yang, University of Nebraska - Lincoln

A.M. Parkhurst, University of Nebraska - Lincoln

T.M. Brown-Brandl, USDA-ARS U.S. Meat Animal Research Center

J.A. Nienaber, USDA-ARS U.S. Meat Animal Research Center

Characterizing Thermal Hysteresis In Body Temperature For A Heat Stressed Steer

F. Yang, University of Nebraska - Lincoln

A.M. Parkhurst, University of Nebraska - Lincoln

D.A. Spiers, University of Missouri - Columbia

J.B. Gaughan, University of Queensland, Gatton, Australia

T.L. Mader, University of Nebraska - Lincoln

G.L. Hahn, US Department of Agriculture (Retired)

Modeling DNA Methylation Tiling Array Data

Gayla R. Olbricht, Purdue University

Bruce A. Craig, Purdue University

R.W. Doerge, Purdue University 
A Generalized Approach and Computer Tool for Quantitative Genetics Study

Jixiang Wu, South Dakota State University

Johnie N. Jenkins, USDA-ARS, Mississippi State

Jack C. McCarty, USDA-ARS, Mississippi State

Using Time-series Intervention Analysis to Model Cow Heart Rate Affected by Programmed Audio and Environmental/Physiological Cues

Dean M. Anderson, U.S. Department of Agriculture, Las Cruces, NM

Norbert Remenyi, Georgia Tech

Leigh Murray, Kansas State University

Generalized Linear Mixed Model Estimation Using Proc Glimmix:

Results from Simulations When the Data and Model Match, and When the Model Is Misspecified

Debbie Boykin, ARS/USDA, Stoneville, MS

Mary J. Camp, ARS/USDA, Beltsville, MD

LuAnn Johnson, University of North Dakota

Matthew Kramer, ARS/USDA, Beltsville, MD

David Meek, NLAE, ARS/USD, Ames, IA

Debra Palmquist, ARS.USDA, Peoria, IL

Bryan Vinyard, ARS/USDA, Beltsville, MD

Mark West, ARS/USDA, Fort Collins, CO

Functional Divergence of Duplicated Genes in the Soybean Genome

Paul L. Auer, Purdue University

R.W. Doerge, Purdue University

On Testing for Significant Quantitative Trait Loci (QTL) Effects When Variances are Unequal

Pradeep Singh, Southeast Missouri State University

Shesh N. Rai, University of Louisville, KY 
Approximate Bayesian Approaches For Reverse Engineering

\section{Biological Networks}

Andrea Rau, Purdue University, INRA AgroParisTech

Florence Jaffrezic, INRA AgroParisTech

Jean-Louis Foulley, INRA AgroParisTech

R.W. Doerge, Purdue University

Nonlinear Regression Parameters As Outcomes: Simple VS.

Sophisticated Analyses

Reid D. Landes, University of Arkansas

A Non-Parametric Empirical Bayes Approach for Estimating Transcript Abundance In Un-Replicated Next-Generation Sequencing Data Sanvesh Srivastava, Purdue University

R.W. Doerge, Purdue University

\section{For Agricultural Dose-Response Experiments \\ M.J. Frenzel, University of Nebraska-Lincoln \\ W.W. Stroup, University of Nebraska-Lincoln \\ E.T. Paparozzi, University of Nebraska-Lincoln}

After Further Review: An Update on Modeling and Design Strategies

\section{Introduction To Selecting Subsets Of Traits For Quantitative Trait 267}

\section{Loci Analysis}

Tilman Achberger, Purdue University

James C. Fleet, Purdue University

David E. Salt, Purdue University

R.W. Doerge, Purdue University 
Achberger, Tilman Ripp, Purdue University Aiken, Rob, Northwest Research-Ext. Center Anderson, Michael, KSU Statistics

Anderson, Steven, Anderson Research, LLC Aref, Susanne, Aref Consulting Group, LLC Bahman, Shafii, University of Idaho Bargen, Jason, Agspan

Baumann, Douglas, Purdue University Bello, Nora, Michigan State University Blankenship, Erin, University of Nebraska Boberg, Wendy, AFMC, MMCS Boyer, John, Kansas State University Boykin, Debbie Leigh, USDA ARS, Statistics Brejda, John, University of Nebraska Brennan, Meghan, University of Florida Brewer, James

Bryson, William, Pfizer Animal Health Camp, Mary, USDA-ARS

Cheng, Fu-Chih, North Dakota State University Chernoff, William, Statistics Cochrane, Chun-Yen, Kansas State University Cui, Zhanglin Lin, Eli Lilly \& Company Dixon, Philip, lowa State University Doerge, Rebecca, Purdue University, Statistics Duke, Sara

Durham, Susan, Utah State University Fabellar, Anabella, Monsanto Company Feng, Lizhi, Pioneer Hybrid International Inc. Fergen, Brian, USDA, APHIS, VA, CVB Statistics Feyerherm, Arlin, Kansas State University Gadbury, Gary, Kansas State University Gbur, Edward, University of Arkansas Green, Jennifer, University of Nebraska-Lincoln Hagel, Emily, University of Nebraska-Lincoln Hassen, Abebe, Pioneer Hybrid International Inc Heaton, Louis, USDA ARS GMPRC Higgins, James, Kansas State University Hinds, Mark, Pioneer Hi-Bred Int'I Hong, Bonnie, Pioneer Hybrid Int'I Huang, Xuewen, Monsanto Company Jiang, Dongming, Monsanto Company Johnson, LuAnn, UND-GFHNRC
Johnson, Dallas, Kansas State University

Jun, Hyoungjin

Kadaru, Suresh, Syngenta Seeds Inc.

Keating, Karen, Kansas State University

Keighley, John, Kansas University Med Center

Kiernan, Kathleen, SAS Institute

Kramer, Matt, USDA ARS

Kratzer, Dal, ESCI

Kreider, Scott, Kansas State University Lamm, Freddie, NW Research Extension Center $\mathrm{Li}$, Xiaopeng, University of Nebraska-Lincoln Liang, Bixia, University of Nebraska - Lincoln Lipka, Alexander Liu, Jia, lowa State University Livermore Auer, Paul, Purdue University Mackey, Bruce, USDA, ARS

MacKinnon, April, Milliken Associates Inc. Meek, David, USDA, ARS Murray, Leigh, Kansas State University Narayan, Sruthi, Kansas State University Neill, Jim, Kansas State University Ochsenfeld,Cherie, Purdue University Olbricht, Gayla, Purdue University Ou, Zhining, Kansas State University Page, Garritt, lowa State University Palmquist, Debra, USDA, ARS Parkhurst, Anne, University of NE - Lincoln Pathak, Manoj, University of NE - Lincoln Peng, Liang, Kansas State University Perera, Muditha, Kansas State University Phillips, Gregory, USDA, NWRC Pintar, Adam, lowa State University Price, Bill, University of Idaho Ramirez, Girly, Kansas State University Ridpath, Harold, Center for Veterinary Biologics Rogers, Paul, Samarakoon, Nishantha, Kansas State University Santen, Edzard Van, Auburn University Scherer, Peter, Dow AgroSciences

Senne, Lane Shehata, Abdrabbo, KSU Research \& Extension Smith, David, New Mexico State University 
Song, Tingting, Kansas State University

St. Pierre, Normand, Ohio State University

Staudhammer, Christina, University of Florida

Stevens, John, Utah State University

Stroup, Walt, University of Nebraska - Lincoln

Thompson, Kevin, University of Arkansas

Tolos, Siti, Kansas State University

Trapp, Allan, lowa State University

Vahl, Christopher, ND State University

VanDyke, Rhonda, University of Cincinnati

Vinyard, Bryan, USDA-ARS

Wang, Dong, University of NE - Lincoln

West, Mark, USDA - ARS

Whitehand, Linda, W Regional Research Center

Wu, Jixiang, Mississippi State University

Xie, Lin, Kansas State University

Yang, Yuqing, Alberta Sustainable Resource Dev.

Yankovskyy, Yevhen, University of Kansas

Yates, Heath, Kansas State University

Yeater, Kathy, USDA - ARS

Yu, Jianming, KSU Dept. of Agronomy

Yum, Man Yu, lowa State University

Zeng, Beiyan, Monsanto Company

Zeng, Yan, University of NE - Lincoln

Zhu, Chengsong, Kansas State University

Zoh, Roger, lowa State University 